\title{
Magic-angle-spinning NMR spectroscopy applied to small molecules and peptides in lipid bilayers
}

\author{
C. Ader, R. Schneider, K. Seidel, M. Etzkorn and M. Baldus ${ }^{\mathbf{1}}$ \\ Solid-state NMR, Max-Planck-Institute for Biophysical Chemistry, Am Fassberg 11, 37077 Göttingen, Germany
}

\begin{abstract}
ssNMR (solid-state NMR) spectroscopy provides increasing possibilities to study the structural and dynamic aspects of biological membranes. Here, we review recent ssNMR experiments that are based on MAS (magic angle spinning) and that provide insight into the structure and dynamics of membrane systems at the atomic level. Such methods can be used to study membrane architecture, domain formation or molecular complexation in a way that is highly complementary to other biophysical methods such as imaging or calorimetry.
\end{abstract}

\section{Introduction}

Biological membranes not only act as isolated barriers but also are heavily involved in the direction and organization of countless cellular processes. ssNMR (solid-state NMR) spectroscopy is a versatile method to study the structural and dynamic aspects of lipid bilayers and molecules associated with them (Figure 1). In general, the term 'solid state' refers to magnetic resonance experiments in which the intrinsic molecular motion is restricted and/or characterized by a molecular correlation time that is comparable with or longer than the inverse of the size of nuclear interactions in the magnetic field. In addition, molecular segments that exhibit fast reorientation in the nanosecond-to-microsecond regime can be monitored, thus enabling ssNMR experiments in a broad range of motional rates.

In general, the advantages of ssNMR compared with other biophysical techniques employed for membrane investigations are the lack of a size restriction for the system to be investigated, the ability to study samples without the introduction of artificial chemical modifications and the option to use samples that are not highly ordered or aligned, if MAS (magic angle spinning; [1]) is performed. In the following, we review selected MAS-based ssNMR experiments that provide insight into the structure and dynamics of membrane systems at the atomic level.

\section{MAS}

In general, the magnitude of the dipolar, the quadrupolar and the anisotropic chemical shielding interaction depends on the orientation of the interaction vector in the static magnetic field. Unless suppressed by the influence of fast isotropic tumbling in solution, a randomly oriented sample

Key words: biological membrane, lipid bilayer, magic angle spinning (MAS), membrane dynamics, small molecule, solid-state NMR.

Abbreviations used: MAS, magic angle spinning; PFG, pulsed field gradients; sSNMR, solid-state NMR.

${ }^{1}$ To whom correspondence should be addressed (email maba@nmr.mpibpc.mpg.de) is hence characterized by a broad ssNMR spectrum. While such spectra have long been analysed in terms of molecular orientation and motion (e.g. using ${ }^{2} \mathrm{H}$ or ${ }^{31} \mathrm{P}$ ssNMR; $[2,3])$, site-resolved information and high-resolution ssNMR spectra are, under such conditions, difficult to obtain. One option to reduce spectral overlap is to study macroscopically aligned membrane samples (see, e.g., [4,5]). However, the preparation of such samples that have been shown to be sensitive to dehydration can be difficult. Alternatively, MAS, where the sample is rapidly spun around an axis tilted by the magic angle $\left(\theta_{\mathrm{m}}=54.7^{\circ}\right)[1,6]$ with respect to the static magnetic field, can be applied. To date, MAS has become the dominant technique for high-resolution ssNMR spectroscopy and can be employed for applications ranging from polymers and microcrystals to lipid bilayers and proteoliposomes.

\section{Localization of small molecules and peptides in lipid bilayers}

More than 20 years ago, Oldfield and co-workers demonstrated that high-resolution ${ }^{1} \mathrm{H}$ spectra of lipid membranes could be obtained under MAS conditions $[7,8]$. Compared with static solids for which ${ }^{1} \mathrm{H}$ signals are generally extensively broadened due to large homonuclear dipolar interactions, the molecular motion of lipids assists the MAS-induced averaging effect and enables high-resolution ${ }^{1} \mathrm{H}$ NMR spectra at comparably low MAS rates $(1-4 \mathrm{kHz}) .{ }^{1} \mathrm{H}$ MAS NMR spectroscopy hence has become the method of choice to investigate the interaction of small molecules with lipid bilayers. This is illustrated in Figure 2, where ${ }^{1} \mathrm{H}$ spectra of asolectin liposomes were recorded for different MAS rates and temperatures. The increased resolution as a result of increased lipid motion or a modest increase in MAS rate is readily apparent.

A straightforward way to study the effect of adding small molecules to lipid membranes is to monitor chemical-shift changes for the lipid resonances in a one-dimensional ${ }^{1} \mathrm{H}$, ${ }^{13} \mathrm{C}$ or ${ }^{31} \mathrm{P}$ MAS NMR spectrum. In the case of phosphorous NMR, not only the isotropic chemical shift but also 
Figure 1 | Possible applications of MAS-based ssNMR spectroscopy on lipid bilayers

Rapid rotation of a solid-state sample around an axis tilted by the magic angle of $\theta_{\mathrm{m}}=54.7^{\circ}$ with respect to the static magnetic field $B_{0}$ is a major technique in ssNMR spectroscopy in order to achieve 'liquid-like' resolution. The spinning frequency $v_{r}$ is typically of the order of several kilohertz. Today, a range of ssNMR experiments is in hand to investigate molecules associated with lipid membranes under MAS. Orientation relative to the membrane bilayer $(\mathbf{A})$, structural characteristics $(\mathbf{B})$, and dynamic properties such as diffusion and intrinsic mobility $(\mathbf{C})$ are features of the molecule of interest that can be explored using MAS NMR spectroscopy.

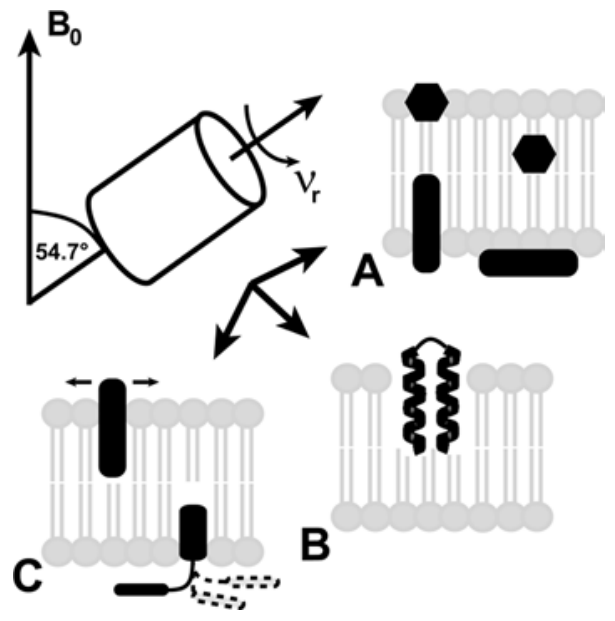

the sideband manifold, generated by the anisotropic chemical shielding interactions, can provide a sensitive means to monitor changes in molecular structure or dynamics due to the presence of a lipid-interacting molecule. A more reliable way to probe spatial proximity via ${ }^{1} \mathrm{H}$ NMR spectro- scopy is NOESY [9] that still forms the basis for protein structure determination in liquid-state NMR spectroscopy. The enhanced resolution observed for spectra of membrane systems under MAS and long spin-lattice relaxation times allow for the acquisition of two-dimensional NOESY spectra. Cross-peak volumes seen in these experiments reflect both inter- and intra-molecular NOESY cross-relaxation rates and hence encode structural and dynamic information. Cross-correlation rates depend on the probability of observing a close spatial proton-proton contact and are sensitive to changes in length and orientation of the vector connecting the interacting protons $[10,11]$.

In order to determine the orientation of peptides or even larger proteins with respect to the bilayer normal in non-oriented samples, experiments that exploit proton spin diffusion have become useful. The faster tumbling rate of free water molecules compared with the protein and the lipid bilayer can, for example, be utilized to selectively use water protons as a polarization source by applying a so-called $\mathrm{T}_{2}$ filter after proton excitation. Such techniques are well established in the context of (bio)polymers and were first utilized in lipid bilayers by Kumashiro et al. [12]. After preparation of $\mathrm{H}_{2} \mathrm{O}$ polarization, a subsequent proton-proton mixing unit leads to polarization of lipid and protein ${ }^{1} \mathrm{H}$ nuclei, which can be read out on dilute spins such as ${ }^{13} \mathrm{C}$ and ${ }^{15} \mathrm{~N}$. Since the rate of transfer depends on the distance between the resulting coupled spins, the magnetization transfer dynamics are sensitive to the spatial orientation of the protein with respect to the source of magnetization. At elevated temperatures above the gel-to-crystalline phase transition for lipids, a similar approach is possible if selective excitation pulses, e.g. weak Gauss-shaped pulses, are introduced. Complementary experiments using lipids or even the protein itself (followed by reversed readout via the lipid and water

Figure $2 \mid{ }^{1} \mathrm{H}$ MAS NMR spectra of asolectin liposomes obtained at MAS frequencies of 1 and $6.5 \mathrm{kHz}$

Left: measured in the liquid crystalline phase at $278 \mathrm{~K}$. Right: measured in the gel phase at $265 \mathrm{~K}$. The rapid axial diffusion of the lipid molecules in conjunction with high-frequency gauche-trons isomerization of the fatty acid chains in the liquid crystalline phase leads to high-resolution proton spectra using a moderate MAS rate. Possible distortions in the spectra at the carrier frequency due to water presaturation are indicated by an asterisk.

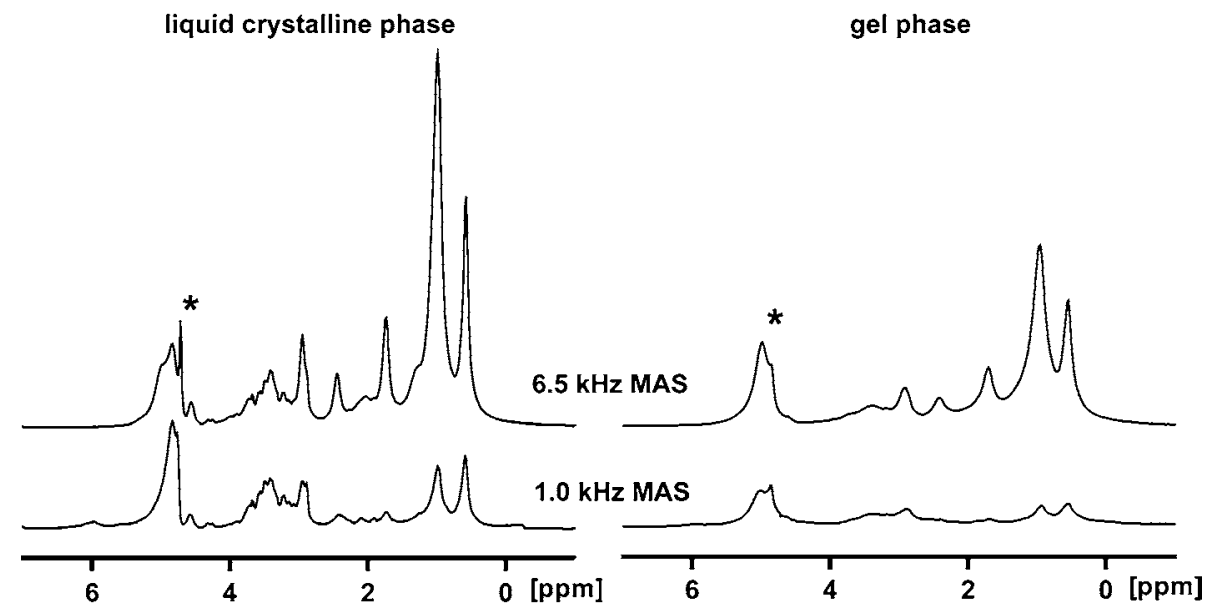


${ }^{1} \mathrm{H}$ NMR signals) as the source of magnetization have been recently introduced by Hong and co-workers [13,14]. In conjunction with numerical simulations of spin diffusion [15] adapted to the system of interest, these experiments are capable of probing peptide orientation in a lipid membrane employing a sample of unaligned multilamellar vesicles under MAS.

As noted earlier, peptide orientations in membranes have long been investigated using macroscopically oriented samples and experiments such as the PISEMA (polarization-inversion-spin-exchange-at-the-magic-angle) experiment [16], which probes the orientation-dependent size of ${ }^{15} \mathrm{~N}-{ }^{1} \mathrm{H}$ dipolar couplings in a two-dimensional ssNMR experiment. Hybrid approaches that employ MAS on macroscopically oriented membranes have provided additional information about the relative orientation of $\alpha$-helical peptides in the lipid environment $[17,18]$. Membrane alignment can be achieved using small glass discs or polymer films (polyetheretherketone), which are then stacked or rolled up inside the MAS rotor. Hence, model bilayers can be constructed that are characterized by a membrane normal orthogonal or parallel to the rotor axis. For membrane-embedded polypeptides oriented on polymer films, a general approach to probe the overall orientation and the local backbone conformation of a polypeptide separately within the same ssNMR scheme can be used [19].

\section{Determination of peptide structure and dynamics in lipid bilayers}

For a long time, ssNMR methods were used to determine specific structural constraints in peptide/liposome preparations, often using selectively labelled peptide variants (see, e.g., $[20,21])$. In order to gain insight into the secondary or tertiary structure of an entire peptide or protein by solidstate NMR spectroscopy, several selectively labelled samples can be used or multiple/uniform $\left({ }^{13} \mathrm{C},{ }^{15} \mathrm{~N}\right)$ isotope labelling is necessary. The resulting polypeptide samples, which can be obtained from solid-phase chemistry or recombinant expression, can be reconstituted into lipid bilayers using established procedures.

First, resonance assignments must be obtained using a combination of ${ }^{15} \mathrm{~N},{ }^{13} \mathrm{C}$ and ${ }^{13} \mathrm{C},{ }^{13} \mathrm{C}$ correlation experiments (see, e.g., [22]). In principle, both through-bond and throughspace interactions can be used in ssNMR. For molecular segments exhibiting motion in the nanosecond-to-microsecond regime, experiments employing scalar couplings that are insensitive to molecular reorientation are the method of choice. In Figure 3, a combined application of through-bond and through-space correlation experiments is shown that delivers sequential resonance assignments of highly mobile and largely immobilized membrane-associated polypeptide segments of the 52-amino-acid protein phospholamban respectively [23]. For the peptide reconstituted in lipid bilayers, the NMR data are consistent with an $\alpha$-helical transmembrane segment and a cytoplasmic domain that exhibits a high degree of structural disorder. Note that the ${ }^{13} \mathrm{C}$ line widths in both protein segments are comparable, underlining the fact that in proteoliposomes the ssNMR line width is not influenced by packing effects.

Once ${ }^{13} \mathrm{C}$ (or, to a lesser degree, ${ }^{15} \mathrm{~N}$ ) resonance assignments have been obtained, these parameters provide a sensitive means of investigating peptide secondary structure. In addition, secondary structure can be investigated by determining internuclear distances under MAS (see, e.g., [2426]) and by correlating two anisotropic interactions such as the CSA (chemical shift anisotropy) and the dipolar coupling in a multidimensional experiment [27]. In order to elucidate the three-dimensional fold of a protein, a larger number of internuclear distance and torsion angle restraints have to be determined. Consecutively, families of molecular conformations are calculated using, for example, simulated annealing Molecular Dynamics procedures, and rated based on experimental results. Complementary approaches are available today for ssNMR-based structure determination, including chemical-shift-selective recoupling techniques $[28,29], \mathrm{CHHC}$ and NHHC two-dimensional experiments that read out proton-proton distances using ${ }^{13} \mathrm{C},{ }^{15} \mathrm{~N}$ COSY [24], and advanced isotope labelling schemes [30,31]. In addition, approaches probing oligomerization in membranes by ssNMR have been developed [32,33]. Furthermore, paramagnetic quenchers [34-36] and deuterium-hydrogen exchange experiments [37] provide additional routes for the analysis of molecular structure in membranes. For a more comprehensive overview of the ssNMR methods discussed in this section, see e.g. [33,38-42].

\section{Diffusion of small molecules and peptides in lipid bilayers}

Since the 1970s, ssNMR spectroscopy has provided insight into the molecular dynamics of membranes (see e.g. [2]). ssNMR methods can probe both local mobility (see the previous section) and the overall correlation time of a molecule in lipid bilayers. For the latter case, PFG (pulsed field gradients) can be combined with MAS-based NMR techniques. Applications show that PFG-MAS NMR spectroscopy allows for the investigation of diffusion in semi-solid environments such as membranes $[43,44]$. Diffusion rates can be determined for molecules with resolved NMR resonances by using gradients in order to dephase and refocus magnetization. If a gradient is applied to transversal magnetization, the Lamor frequency becomes dependent on the location of the individual spin in the gradient. For a sufficiently strong gradient, the transversal magnetization will therefore be uniformly distributed and no NMR signal will be detected. Application of an inverse gradient can refocus the transversal magnetization and hence recover the NMR signal. If, however, the molecule underwent rotational or translational diffusion during the delay time, magnetization will not be refocused completely and the resulting signal will be attenuated. Analysis of the NMR signal modulation allows one to determine the diffusion coefficient for a given motional 
Figure 3 Results of an HC-INEPT (through-bond) experiment (upper panel) and a CC double-quantum/single-quantum correlation experiment (lower panel) on a uniformly labelled sample of monomeric phospholamban

The indicated resonance assignments and further experimental conditions are given in [23]. Contributions from natural abundance lipid background are marked with an asterisk.
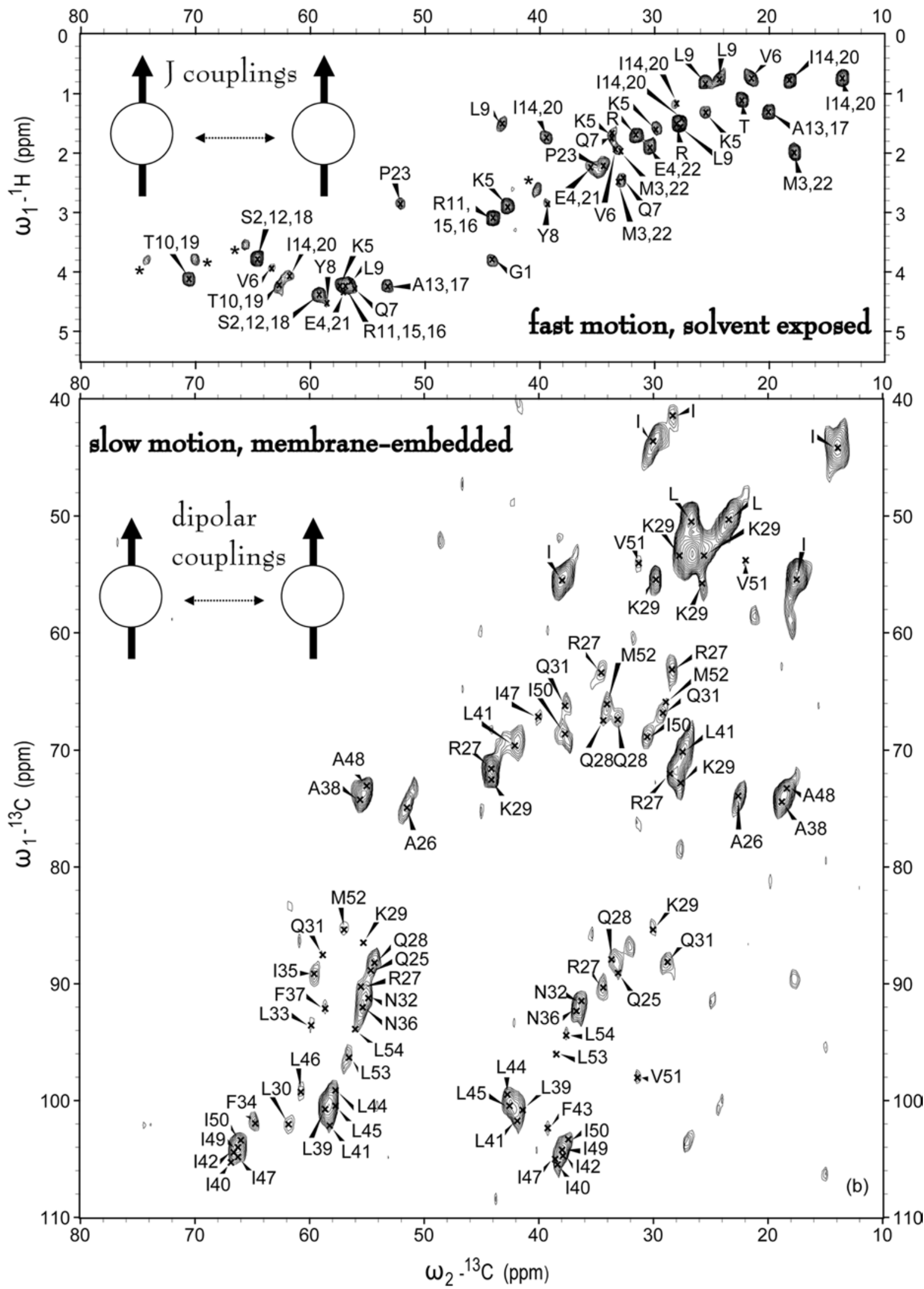
model. Generally, smaller length scales and shorter diffusion delays require stronger gradients.

\section{Summary}

In this review, we have briefly described the versatile use of NMR methods to study lipid structure and dynamics and lipid-small molecule interactions under MAS conditions. Of course, it was not possible to appropriately consider the entire research field, and the interested reader is strongly encouraged to use references given here as a starting point for further study. In general, experiments such as those described here can not only be applied to study the structure and dynamics of membrane systems of increasing size and complexity $[33,38-42,45]$ but they also readily allow for probing the effect of changes in lipid composition, temperature or ion concentration at the atomic level. Methods described here hence can be used in applications ranging from membrane biophysics to drug discovery in a way that is highly complementary to other biophysical methods such as imaging or calorimetry.

Discussions with Dr Henrike Heise and Dr Stefan Becker are gratefully acknowledged. Work described here was funded in part by the DFG (Deutsche Forschungsgemeinschaft) and the Max Planck Society. R.S. thanks the DFG graduate school 782 'Spectroscopy and dynamics of molecular aggregates, chains and coils' for a Ph.D. fellowship. C.A. acknowledges financial support from the $\mathrm{FCl}$ (Fonds der Chemischen Industrie).

\section{References}

1 Andrew, E.R., Bradbury, A. and Eades, R.G. (1958) Nature 182, 1659

2 Seelig, J. (1977) Q. Rev. Biophys. 10, 353-418

3 Seelig, J. (1978) Biochim. Biophys. Acta 515, 105-140

4 Cross, T.A. and Opella, S.J. (1994) Curr. Opin. Struct. Biol. 4, 574-581

5 Opella, S.J., Nevzorov, A., Mesleh, M.F. and Marassi, F.M. (2002)

Biochem. Cell Biol. 80, 597-604

6 Lowe, I.J. (1959) Phys. Rev. Lett. 2, 258-287

7 Forbes, J., Bowers, J., Shan, X., Moran, L., Oldfield, E. and Moscarello, M.A. (1988) J. Chem. Soc. Faraday Trans. 84, 3821-3849

8 Forbes, J., Husted, C. and Oldfield, E. (1988) J. Am. Chem. Soc. 110 1059-1065

9 Jeener, J., Meier, B.H., Bachmann, P. and Ernst, R.R. (1979) J. Chem. Phys. 71, 4546-4553

10 Ernst, R.R., Bodenhausen, G. and Wokaun, A. (1987) Principles of Nuclear Magnetic Resonance in One and Two Dimensions, Clarendon Press, oxford
11 Gawrisch, K., Eldho, N.V. and Polozov, I.V. (2002) Chem. Phys. Lipids 116, 135-151

12 Kumashiro, K.K., Schmidt-Rohr, K., Murphy, III, O.J., Ouellette, K.L., Cramer, W.A. and Thompson, L.K. (1998) J. Am. Chem. Soc. 120, 5043-5051

13 Huster, D., Yao, X. and Hong, M. (2002) J. Am. Chem. Soc. 124, 874-883

14 Luo, W. and Hong, M. (2006) Solid State Nucl. Magn. Reson. 29, 163-169

15 Schmidt-Rohr, K. and Spiess, H.W. (1994) Multidimensional Solid-state NMR and Polymers, Academic Press, London

16 Wu, H.C., Ramamoorthy, A. and Opella, S.J. (1994) J. Magn. Reson. Ser. A 109, 270-272

17 Glaubitz, C. and Watts, A. (1998) J. Magn. Reson. 130, 305-316

18 Sizun, C. and Bechinger, B. (2002) J. Am. Chem. Soc. 124, 1146-1147

19 Andronesi, O.C., Pfeifer, J.R., Al-Momani, L., Özdirekcan, S., Rijkers, D.T.S., Angerstein, B., Luca, S., Koert, U., Killian, J.A. and Baldus, M. (2004) J. Biomol. NMR 30, 253-265

20 McDowell, L.M. and Schaefer, J. (1996) Curr. Opin. Struct. Biol. 6, 624-629

21 Bodner, M.L., Gabrys, C.M., Parkanzky, P.D., Yang, J., Duskin, C.A. and Weliky, D.P. (2004) Magn. Reson. Chem. 42, 187-194

22 Baldus, M. (2002) Prog. Nucl. Magn. Reson. Spectrosc. 41, 1-47

23 Andronesi, O.C., Becker, S., Seidel, K., Heise, H., Young, H.S. and Baldus, M. (2005) J. Am. Chem. Soc. 127, 12965-12974

24 Lange, A., Seidel, K., Verdier, L., Luca, S. and Baldus, M. (2003) J. Am. Chem. Soc. 125, 12640-12648

25 Todokoro, Y., Yumen, I., Fukushima, K., Kang, S.-W., Park, J.-S., Kohno, T., Wakamatsu, K., Akutsu, H. and Fujiwara, T. (2006) Biophys. J. 91, 1368-1379

26 Seidel, K., Etzkorn, M., Heise, H., Becker, S. and Baldus, M. (2005) ChemBiochem 6, 1638-1647

27 Rienstra, C.M., Hohwy, M., Mueller, L.J., Jaroniec, C.P., Reif, B. and Griffin, R.G. (2002) J. Am. Chem. Soc. 124, 11908-11922

28 Nomura, K., Takegoshi, K., Terao, T., Uchida, K. and Kainosho, M. (1999) J. Am. Chem. Soc. 121, 4064-4065

29 Jaroniec, C.P., Tounge, B.A., Herzfeld, J. and Griffin, R.G. (2001) J. Am. Chem. Soc. 123, 3507-3519

30 Lemaster, D.M. and Kushlan, D.M. (1996) J. Am. Chem. Soc. 118 9255-9264

31 Castellani, F., van Rossum, B., Diehl, A., Schubert, M., Rehbein, K. and Oschkinat, H. (2002) Nature 420, 98-102

32 Mani, R., Cady, S.D., Tang, M., Waring, A.J., Lehrer, R.I. and Hong, M. (2006) Proc. Natl. Acad. Sci. U.S.A. 103, 16242-16247

33 Hong, M. (2006) Structure 14, 1731-1740

34 Brown, L.R., Braun, W., Kumar, A. and Wüthrich, K. (1982) Biophys. J. 37 319-328

35 Villalain, J. (1996) Eur. J. Biochem. 241, 586-593

36 Tuzi, S., Hasegawa, J., Kawaminami, R., Naito, A. and Saito, H. (2001) Biophys. J. 81, 425-434

37 Tian, C.L., Gao, P.F., Pinto, L.H., Lamb, R.A. and Cross, T.A. (2003) Protein Sci. 12, 2597-2605

38 McDermott, A.E. (2004) Curr. Opin. Struct. Biol. 14, 554-561

39 de Groot, H.J.M. (2000) Curr. Opin. Struct. Biol. 10, 593-600

40 Griffin, R.G. (1998) Nat. Struct. Biol. 5, 508-512

41 Opella, S.J. and Marassi, F.M. (2004) Chem. Rev. 104, 3587-3606

42 Baldus, M. (2006) Curr. Opin. Struct. Biol. 16, 618-623

43 Pampel, A., Michel, D. and Reszka, R. (2002) Chem. Phys. Lett. 357, $131-136$

44 Gaede, H.C. and Gawrisch, K. (2004) Magn. Reson. Chem. 42, 115-122

45 Etzkorn, M., Martell, S., Andronesi, O.C., Seidel, K., Engelhard, M. and Baldus, M. (2007) Angew. Chem. Int. Ed. Engl. 46, 459-462

Received 22 June 2007

doi:10.1042/BST0350991 